\title{
Impact of sleep duration and quality on obesity and diabetes
}

\author{
Daniela C. S. Baldan (IC), Mariana B. Boiani (IC), Aglecio L. Souza (PG), Elinton A. Chaim (PQ), \\ Elizabeth J. Pavin (PQ), Liane M. Rocha (PG), Sarah Monte Alegre (PQ) \\ Abstract \\ There is a link between sleep and obesity and also insulin resistance. We evaluated the relationship \\ between sleep deprivation and the quality of sleep in the the metabolic profile in diabetic obese.
}

Key words: sleep duration, sleep quality, diabetes.

\section{Introduction}

The inverse relationship between the rise of obesity and the restriction of hours of sleep is known. There is also a link between sleep and insulin resistance. In what way this restriction and sleep quality may influence the metabolic profile in diabetic obese?

To establish the relationship between sleep deprivation and the quality of sleep, biochemical changes and anthropometric parameters of normal weight, obese and diabetic obese individuals.

\section{Results and Discussion}

We evaluated 108 adult patients (82 women), divided in three groups: normal weight (NW, $\mathrm{n}=31)$, obese $(\mathrm{Ob}, \mathrm{n}=47)$ and diabetic obese (ObDM, $\mathrm{n}=30$ ). The average age and BMI were: NW $33,7 \pm 10,8$ and $22,3 \pm 1,9$; Ob $40,4 \pm 11,2$ and $38,2 \pm 7,7$; ObDM $44,1 \pm 9,2$ years old and $41,8 \pm 8,1$ $\mathrm{kg} / \mathrm{m} 2$. The diabetics making use of oral drugs for DM control. Sleep quality was assessed by the Pittsburg Sleep Quality Index (PSQI) and drowsiness by Epworth Sleepiness Scale. The clinical and anthropometric assessment included: blood pressure (BP), weight, height, BMI, waist circumference (WC) and body fat (\%BF) by tetrapolar bioimpedance. Biochemical tests: glucose, insulin, $\mathrm{HbA1c}$, total cholesterol, HDL-C, LDL-C, VLDL, triglycerides (TG). Insulin resistance was calculated by the HOMA-IR index. Data were analyzed using SPSS v.20, used the Kruskal-Wallis test and Spearman correlation, $p<$ 0.05 .

Groups didn't differ in: time to go to bed and getting up, latency, average of sleep hours and day sleepiness. There was difference to the sleep quality score among NW and $\mathrm{Ob}(4,3 \pm 3,3 \mathrm{e}$ $8,0 \pm 4,5)$; NW e ObDM $(7,2 \pm 5,3 ; p=0,008)$. In ObDM group the sleep quality was correlated to the lean mass $(r=-0,45 ; p=0,02)$ and $\mathrm{HbA1c}(r=$ 0,$37 ; p=0,05$ ), time to go to bed was correlated to blood glucose level ( $r=0,40 ; p=0,03)$. Groups were divided according to hours of sleep: short sleep $(<6 h)$ and normal sleep $(>6 h)$. There was difference \%BF $(40,5 \pm 11,4$ vs $34,6 \pm 10,5 \% ; p=$ $0,01)$, \% lean mass $(59,4 \pm 11,4$ vs $65,4 \pm 10,5 \% ; p=$ $0,01)$, HbA1c $(6,0 \pm 1,1$ vs $5,6 \pm 0,9 \% ; p=0,02)$ and blood glucose level $(96,7 \pm 23,4$ vs $90,2 \pm 25,4$ $\mathrm{mg} / \mathrm{dL} ; \mathrm{p}=0,01$ ), respectively.

\section{Conclusions}

Sleep quality can be a risk factor to decrease lean mass and increase $\mathrm{HbA} 1 \mathrm{c}$, and the time to go to bed to increase the fasting glucose. The greater diferences are related to sleep duration. Those who sleep less have higher $\% \mathrm{BF}$, less lean mass, high levels of $\mathrm{HbA1c}$ and fasting glucose. In clinical practice, subjects should be evaluated for the presence of sleep disturbance in order to design a more effective treatment to obesity and DM2.

\section{Acknowledgement}

There are no conflicts of interest. 